\title{
CoRrEction OF SATURATED REgIONS IN RGB COLOR SPACE
}

\author{
Hae Jin Ju, Rae-Hong Park \\ Department of Electronic Engineering, School of Engineering, Sogang University \\ 35 Baekbeom-ro (Sinsu-dong), Mapo-gu, Seoul 04107, Korea
}

\begin{abstract}
In a digital image, color representation of a digital image sensor is limited to a narrow dynamic range. Especially, when extremely bright light is captured, the original color of a scene is saturated to the maximum value, up to which a digital image sensor can represent the color. This paper proposes an algorithm that corrects the color in a saturated region, where the original color is distorted and lost. For natural correction, i.e., to minimize the artifacts near the boundary of a saturated region, the proposed method uses the weighted sum of color value(s) in the saturated color channel(s) of neighborhood of saturated regions. In determining the weight of each pixel, saturation, hue, and color values are used with the certainty map. Using the certainty map, the proposed method can reliably distinguish the unsaturated and already desaturated neighboring pixels from the remaining pixels. Then, the proposed correction method computes the weight function using saturation, hue, and color values. Therefore, the proposed algorithm can get reliable corrected colors. Comparison of experimental results of the proposed and existing correction methods shows the effectiveness of the proposed saturated region correction method in the view of natural color restoration.
\end{abstract}

\section{KEYWORDS}

Color saturation, Color restoration, Color correction, Saturated region, Dynamic range, Color artifacts, Weighted sum

\section{INTRODUCTION}

Color digital image mostly has the limited dynamic range in representing color. Thus, when the light passed through the lens is extremely bright, the original color of an object or scene is not represented correctly in a digital image. If the color value of light exceeds the maximum value, up to which an image sensor visualizes the color, the corresponding color value is clipped to the maximum value. In such a case, the clipped pixel is said to be saturated. In addition, the surrounding pixels influenced by saturated pixels also lose their colors, in which the values of just one or two color channels are saturated or have the values close to the maximum color value. These pixels are also said to be saturated. Therefore, the saturated pixels, which have wrong color values, need to be corrected in order to represent the original color value faithfully.

In a digital image, saturation occurs due to the fact that color representation of light passed through a digital image sensor is limited to the low dynamic range [1] . To recover the color from saturation, several existing methods [2] , [3] proposed the color correction algorithms, but they were limited to one-dimensional signals or constrained to the trained set of colors. However, various methods for correcting the saturated regions were proposed through the characteristic analysis of color digital images. Perez et al.'s method [4] corrected the specular reflection or under-exposure using Poisson image editing, which can be applied to local illuminance changes. Meylan et al.'s method [5] removed specular highlight by estimating the brightness in the

DOI : 10.5121/ijcga.2016.6201 
clipped regions. However, the color restoration result is not obtained since estimation of color is not performed on the clipped regions in this method. Tan et al.'s method [6] and Shen and Cai's method [7] also removed specularity in highlighted regions, but could not recover the real scene with original light. All the methods previously mentioned proposed various color correction algorithms, with analyzing the characteristics of specular highlight contained in an image.

Mairal et al.'s method [8] proposed the restoration method of an image containing color saturation. However, this method is not proper to desaturate a digital image because of the restriction to solving the problem in the view of noise reduction. Zhang and Brainard [9] proposed a Bayesian algorithm, which estimates the color in the saturated region. This method used the cross-correlation between the colors in the saturated and non-saturated channels. Masood et al. [10] classified an image into three types of regions: non-saturated, partially saturated, and totally saturated. In partially saturated region, one or two color channels are saturated, whereas in totally saturated region, all three color channels (R, G, and B) are saturated. Then, in order to desaturate an image, they estimated the color ratios by solving two separate cost functions. Guo et al.'s method [11] detected over-exposure in an image, then defined overexposed map. To correct over-exposure, they recovered lightness and color by optimizing respective energy functions. Rouf et al. [12] estimated hue values in the saturated regions, and then restored the color values using estimated hue value and gradient information in the nonsaturated channel(s) of a saturated region. Mansour et al.'s method [13] used a hierarchical windowing algorithm, which divides an image into nonoverlapping $\left(2^{l+1}\right) \times\left(2^{l+1}\right)$ blocks according to the hierarchical level $l$, to detect and correct a saturated region. Only in the block, where the percentage of the number of clipped pixels is smaller than a predefined threshold, this method corrected a saturated pixel using weighted $l_{1}$ minimization, smoothing, and scaling. In Elboher and Werman's method [14], after several steps of bilateral filtering [15] , [16], the saturated regions were determined as the regions with the intensity value larger than the threshold and with gradient norm in the top 10 percentile. After that, in each saturated color cluster, color lines are corrected by stretching the lines if the property that color lines are smooth, elongated, and positively correlated is not satisfied. Xu et al.'s method [17] selected the saturated region by thresholding after bilateral filtering [15] , [16] in RGB color space. Then, after converting color space from RGB to $\mathrm{C}_{\mathrm{b}} \mathrm{C}_{\mathrm{r}}$ channels, both $C_{b}$ and $C_{r}$ values are corrected by convolution using a Gaussian filter. To reduce unnaturalness between the corrected and non-saturated regions, converted $R, G$, and $B$ values are smoothed.

Existing methods used bilateral filtering [15] , [16] for detecting the saturated regions with high reliability [14] , [17] , [18] . Additionally, the lost color values caused by saturation are corrected by considering the correlations among RGB color channels [8] , [14] , [17] , [18] . The saturated regions are corrected well by applying the correlation between color channels, but most existing methods used complex methods such as convolution or optimization to correct the color values. Thus, our proposed algorithm corrects the saturated regions using a relatively simple computation method, which uses the weighted sum of the color values of unsaturated neighboring pixels of a saturated region. Without additional post-processing, the corrected results with natural color representation, in the boundaries between the saturated and non-saturated regions, can be obtained by the weighted sum. In calculating the weight function used in a weighted sum, certainty map, $R, G$, and $B$ color, saturation, and hue values are used. Additionally, to increase the reliability of the pixels used in correction process, the proposed method excludes the saturated pixels by using the certainty map [17] . In addition, by using hue values as well as RGB color values in weighting functions, the correlation among RGB color channels is also recovered.

The rest of the paper is organized as follows. In Section 2, the method for detecting the saturated region [17] is described. Then, in Section 3, the proposed method for correcting color saturation is explained. The proposed method is performed using the weighted sum of color values in the 
neighborhood of a saturated region. Experimental results and discussions are shown in Section 4. Finally, in Section 5, conclusions are given.

\section{SATURATion Region Detection}

The saturated region in an image is detected using the detection method used in Xu et al.'s methods [17] , [18] . The pixel, which has the color value exceeding saturation threshold $T_{\text {sat }}$, e.g., '235' in an 8-bit image [10], [19] , is classified as the saturated pixel. If at least one of $R, G$, and $B$ values at a pixel exceeds $T_{s a t}$, then this pixel belongs to the saturated region. Then, the pixel value of the saturated color channel is only corrected. Threshold $T_{\text {sat }}$ is determined according to the characteristics of charge-coupled device (CCD) [19] . If bright light is passed through CCD or complementary metal-oxide-semiconductor sensor during exposure time, the charges collected in a cell defining a single pixel overflow potential well [20], [21] . In such a case, the corresponding pixel is saturated. Exact saturation threshold value can be changed according to the characteristics of various sensors, but $T_{\text {sat }}$ can be decided as ' 235 ' in a full resolution (8-bit) image because of the nonlinear characteristics of CCD sensor [19] .

Prior to classifying the saturated pixels in each of the R, G, and B color channels using threshold $T_{\text {sat }}$, existing methods [14] , [17] , [18] used bilateral filtering [15] , [16] in order to reduce noise. A bilateral filter eliminates an isolated saturated region or a hole caused by noise within the saturated region, preserving the edges in an image. In addition, a variety of in-camera image processing steps (such as camera response, sensor noise, color filter array interpolation, etc.) make the pixels have smaller values although the original pixels have the maximum color values [17] . Insufficient detection of saturated region caused by the reasons mentioned previously can be also prevented by using a bilateral filter. After bilateral filtering, all R, G, and B channels are independently thresholded using $T_{\text {sat }}$ to detect the saturated regions. That is, the pixel belongs to the saturated region if when at least one value among $R, G$, and $B$ color values is larger than $T_{\text {sat }}$.

Figure 1 shows the difference of the saturated regions with and without bilateral filtering applied to an input image [17] . In Figures 1(a) and 1(b), the input image without bilateral filtering [15], [16] and the resulting image of the detected regions with Figure 1(a) are shown, respectively. Figures 1(c) and 1(d) show the image with bilateral filtering and the resulting image of the detected regions with Figure 1(c), respectively. Especially, Figures 1(b) and 1(d) are the cropped images indicated by red boxes in Figures 1(a) and 1(c), respectively, in which colored and monochrome representations are used in the saturated and non-saturated regions, respectively. In Figure 1(b), some saturated regions are constructed by a single or a couple of pixels. Additionally, there are small holes in the wide saturated region. On the other hand, in Figure 1(d), most isolated regions and holes are removed compared to the image in Figure 1(b). As a result, the proposed method does not need to perform correction procedure on actually nonsaturated regions, in which saturation is caused by noise in an image.

In a bilateral filtered image, the saturated regions are partitioned and labeled in order to separate one region from the others [17] . That is, if two regions lie apart in an image, then each region is labeled differently. After that, correction is performed in each saturated region with different label. 


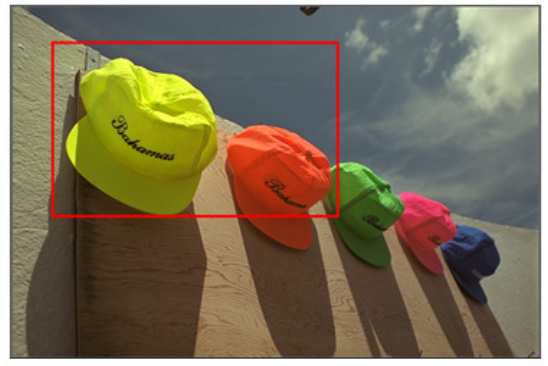

(a)

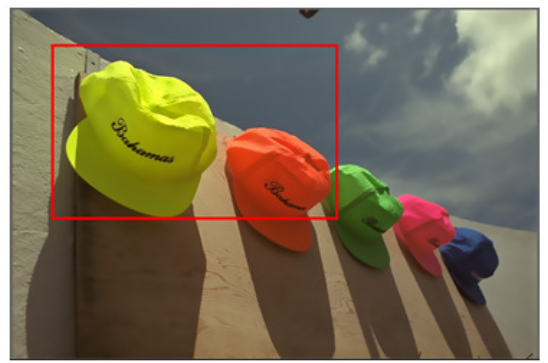

(c)

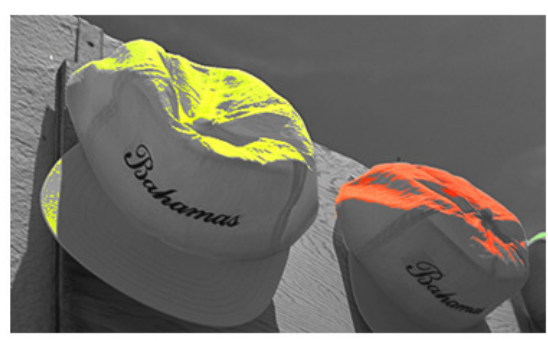

(b)

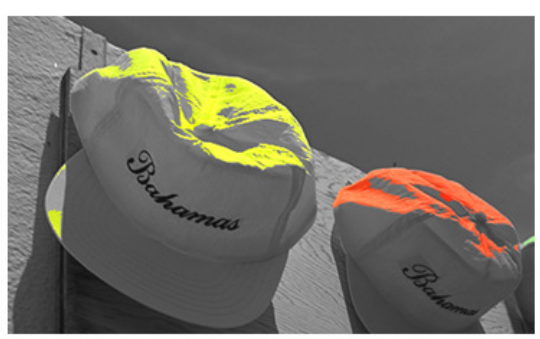

(d)

Figure 1. Detection of the saturated region. (a) Input image, (b) Detected saturated region of (a), represented with color, (c) Bilateral filtered image of (a), (d) Detected saturated region, represented with color, of bilateral filtered image.

\section{Proposed Saturated Region Correction Method}

In this section, the proposed method, which corrects the pixels in the saturated region using the weighted sum of color values at neighboring pixels, is described. To explain how the proposed method composes and uses the weight function, in Section 3.1, we present the correction method using weighted sum. Then, three sub-weight functions in order to calculate weight functions are explained in Section 3.2.

\subsection{Proposed Correction Method Using Weighted Sum}

The proposed method corrects the pixels in the saturated region using the weighted sum of each color value at unsaturated neighboring pixels. These saturated pixels are corrected in each saturated channel independently. For example, if a pixel is saturated in both $\mathrm{R}$ and $\mathrm{G}$ channels, then $R(G)$ value is corrected using only $R(G)$ values of neighboring pixels. Thus, the correction of each saturated color channel in a saturated region is performed as

$$
\hat{C}(x, y)=\frac{1}{\sum_{i, j \in M} W_{x+i, y+j}} \sum_{i, j \in M} W_{x+i, y+j} C(x+i, y+j),
$$

where $C$ and $\hat{C}$ are the original and the corrected color values, respectively, $W_{x+i, y+j}$ is the weight at pixel $(x+i, y+j)$, and $M$ represents the $(2 k+1) \times(2 k+1)$ window centered at saturated pixel $(x, y)$. Note that $(x+i, y+j)$ denotes the pixel in the neighboring window of $(x, y)$.

In correcting the color values of each saturated region using (1), the correction procedure is applied first to the pixels with smaller distance from the nearest neighboring pixel that is not 
saturated [17] . To satisfy this constraint, morphological erosion and logical AND operations [22] , [23] , [24] are used in the proposed method. In Figure 2, the procedure, which determines which pixel is first corrected in each saturated region with different label, is shown. Figure 2(a) shows the image with colored saturated region with label ' 7 '. Figure 2(b) is the binary image, in which only the saturated region is indicated with the value ' 1 ', and Figure 2(c) is the image eroded from Figure 2(b). The image of Figure 2(c) is obtained from AND operation with Figure 2(b) and inverted image of Figure 2(c), i.e., the values ' 0 ' and ' 1 ' of Figure 2(c) are interchanged. Through the procedure from Figures $2(\mathrm{~b})$ to $2(\mathrm{~d})$, the most outer region in the saturated region is detected as shown in Figure 2(d). Then, the second most outer region, which is represented by the value ' 1 ' in the binary image of Figure $2(\mathrm{e})$, is obtained using the binary image of Figure 2(c) and the eroded image from Figure 2(c). Figure 2(f) is obtained through the same procedure. That is, from Figures 2(d) to 2(f), the iteration of morphology and AND logical operation is carried out to correct the saturated pixels in order of decreasing distance between the pixels in the saturated region and the non-saturated neighboring pixels. Iteration is performed until there is no remaining pixel detected. As a result, the correction is performed on the pixels with the value ' 1 ' in Figures 2(d) to 2(f), in consecutive order. According to this correction order, the proposed method can get reliability of the weight function which is related to the certainty map [17] .

In calculating (1), the weight $W_{x+i, y+j}$ is determined using the three weight functions, i.e., $W_{x+i, y+j}^{C}, W_{x+i, y+j}^{S}$, and $W_{x+i, y+j}^{H}$, as

$$
W_{x+i, y+j}=W_{x+i, y+j}^{C} \times W_{x+i, y+j}^{S} \times W_{x+i, y+j}^{H}
$$

where $W_{x+i, y+j}^{C}, W_{x+i, y+j}^{S}$, and $W_{x+i, y+j}^{H}$ are the weights related to the certainty map [17] and color, saturation, and hue [22], respectively, and the detailed explanation will be given in Section 3.2.

\subsection{Weight Functions}

The proposed method corrects saturated regions using weighted sum of non-saturated or desaturated neighboring pixels as in (1). The three weight functions, $W_{x+i, y+j}^{C}, W_{x+i, y+j}^{S}$, and $W_{x+i, y+j}^{H}$, at each pixel are determined by considering the certainty map [17] and color, saturation, and hue [22] . The neighboring pixels that are already saturated should not be used in correction process, thus the proposed method uses the certainty map and combines color property in the weight function. On the other hand, if the neighboring pixel is not saturated or already desaturated, the weight is given according to the difference of color values of the corresponding pixel and the saturated pixel. In addition, the neighboring pixels to be used in the weighted sum should have low saturation and hue similar to the saturated region for reliable correction. 


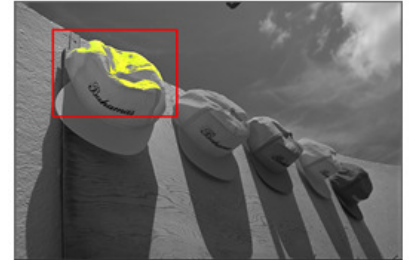

(a)

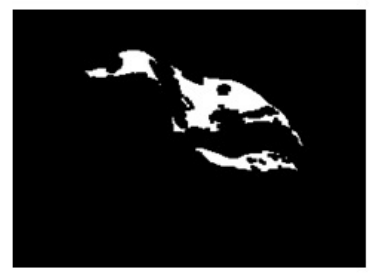

(c)

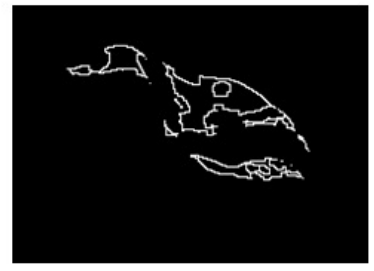

(e)

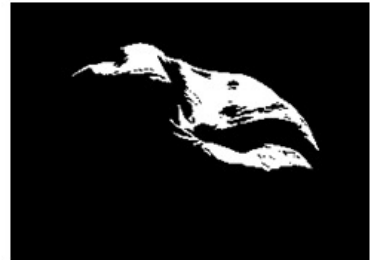

(b)

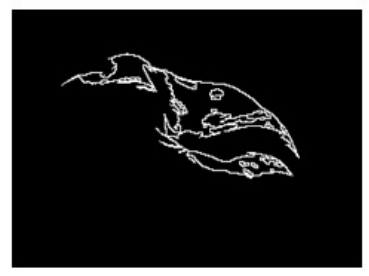

(d)

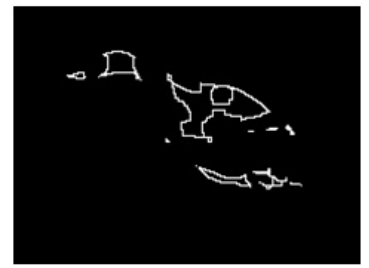

(f)

Figure 2. Detection procedure of the first corrected saturated pixels. (a) Detected saturated region with label ' 7 ' and represented with color, (b) Binary image of (a), where colored region is represented by ' 1 ' and the others ' 0 ', (c) Morphological eroded image of (b), (d) Most outer region of (b), which has the value '1', (e) Pixels that are corrected next to the pixels with the value ' 1 ' in (d), (f) Pixels that are corrected next to the pixels with the value ' 1 ' in (e).

Among the pixels in the neighboring region, the saturated pixel should be excluded in calculating the corrected pixel value because of unreliability of the saturated pixel. The color values of saturated pixels are not proper to be used in correction process since their color values do not accurately represent real colors of a scene, thus these pixels should be also corrected. Therefore, the weight of the corresponding pixel should be ' 0 ' by the certainty map [17]. However, in correction process, the desaturated color values, which have been already corrected, of saturated pixels can be used. In this case, the corrected color values of the corresponding pixel are reliable to be referenced in calculating the desaturated color values of the saturated pixel. It is because that the desaturated values are modified to have the colors close to the original colors of a scene. As a result, we use the weight which is similar to the difference of color values in a bilateral filter [15] , [16] with the certainty map. In a bilateral filter, it is noted that a range weight is expressed using a Gaussian function of the color differences between the center and neighboring pixels. Similarly, the proposed method defines the color weight using a Gaussian function of the saturated color channel. The color differences between the saturated and neighboring pixels are used for a Gaussian function, in which the smaller the color differences are, the more similar the colors of neighboring pixels and the original colors of the saturated pixel are. Thus, the color weight function $W_{x+i, y+j}^{C}$ related to the certainty map and the color difference is described as 


$$
W_{x+i, y+j}^{C}(i, j)= \begin{cases}e^{-\frac{1}{2} \frac{C(x, y)-\left.C(x+i, y+j)\right|^{2}}{\sigma_{C}{ }^{2}}}, & \text { if } C(x+i, y+j)<T_{\text {sat }} \\ e^{-\frac{1}{2} \frac{C(x, y)-\left.\hat{C}(x+i, y+j)\right|^{2}}{\sigma_{C}{ }^{2}}}, & \text { if } C(x+i, y+j)>T_{\text {sat }} \text { and } \hat{C}(x+i, y+j)<C(x+i, y+j), \\ 0, & \text { otherwise }\end{cases}
$$

where photometric spread $\sigma_{C}$ determines how the neighboring pixel is weighted by the color difference between the pixels $(x, y)$ and $(i, j)[15] . W_{x+i, y+j}^{C}$ is defined as three values according to the constraints in (3). As previously mentioned, if the color value $C(x+i, y+j)$ is smaller than $T_{s a t}, W_{x+i, y+j}^{C}$ is given as a Gaussian function of the color difference between $C(x, y)$ and $C(x+i$,

$y+j)$. However, if $C(x+i, y+j)$ exceeds $T_{s a t}$ and the desaturation procedure is applied to pixel $(x+i$, $y+j), W_{x+i, y+j}^{C}$ is defined as a Gaussian function using the color difference between $C(x, y)$ and $\hat{C}(x+i, y+j)$ as parameter. Otherwise, $W_{x+i, y+j}^{C}$ is defined as ' 0 ' because of the unreliability, i.e., neighboring pixel is also saturated and not desaturated.

At each neighboring pixel, the proposed method classifies two types of weights using hue $(H)$ and saturation $(S)$ values. Hue and saturation have the characteristics that distinguish one color from another [22] . Besides $R, G$, and $B$ values, hue needs to be used to determine the color similarity between the neighboring pixels and the saturated pixel because predominant color property is represented by $H$ value. In addition, saturation should be also used to compute the weight $W_{x+i, y+j}$ since saturation shows the property about the degree of white light mixed with a specific hue. Note that in order to calculate the weights; $H$ and $S$ values are converted from $R, G$, and $B$ values [22] as

$$
\begin{aligned}
& H= \begin{cases}\frac{\theta}{360}, & \text { if } B \leq G \\
\frac{360-\theta}{360}, & \text { if } B>G,\end{cases} \\
& \theta=\cos ^{-1}\left\{\frac{\frac{1}{2}[(R-G)+(R-B)]}{\sqrt{(R-G)^{2}+(R-B)(G-B)}}\right\}, \\
& S=1-\frac{3}{(R+G+B)}[\min (R, G, B)],
\end{aligned}
$$

where $R, G$, and $B$ are scaled to the range from 0 to 1 . In (4) and (6), both $H$ and $S$ are in the range $[0,1] . H$ in (4) is calculated using $\theta$ in (5) and is defined in two cases according to the relation of $B$ and $G$ values because the inverse of cosine function results in $[0,180]$ (degree). If $H$ value, which has dominant color property, of the neighbouring pixel is much different from that of the saturated pixel, $H$ value is not appropriate to use for recovering the original color of the saturated pixel. Then, the corresponding pixel should have a weight close to ' 0 '. Additionally, when the extent of saturation in the neighboring pixel is large, the possibility that color of the corresponding pixel is distorted is high. In (6), $S$ value becomes small when a pixel is largely saturated. For example, $S$ value is ' 0 ' if all of $R, G$, and $B$ values are '255'. Thus, the weight at 
the neighboring pixel should be proportional to $S$ value in (6). According to the properties of hue and saturation, the proposed method uses the following two weight functions of $H$ and $S$ [17] as

$$
\begin{gathered}
W_{x+i, y+j}^{H}=e^{-\frac{|H(x, y)-H(x+i, y+j)|^{2}}{\sigma_{H}^{2}}}, \\
W_{x+i, y+j}^{S}=1-e^{-\frac{S(x+i, y+j)^{2}}{\sigma_{S}^{2}}},
\end{gathered}
$$

where $\sigma_{H}$ and $\sigma_{S}$ perform the same role as $\sigma_{C}$ in (3) for the hue difference and saturation, respectively. In defining hue and saturation weight functions, the proposed method has a Gaussian form as in a color weight function of (3). Therefore, the resulting weight values of $W_{x+i, y+j}^{H}$ and $W_{x+i, y+j}^{S}$ lie in the range [0,1]. In (7), by using the absolute hue difference as an exponent, $W_{x+i, y+j}^{H}$ has a large value if the hue difference is small. $W_{x+i, y+j}^{S}$ is defined as in (8) since the smaller the degree of saturation is, the smaller $S$ value is. As a result, the weight at each neighboring pixel is determined as the product of the values of $W_{x+i, y+j}^{C}, W_{x+i, y+j}^{S}$, and $W_{x+i, y+j}^{H}$ as in (2).

\section{EXPERIMENTAL RESULTS AND DISCUSSIONS}

Experiments for evaluating the performance of the proposed method are shown using various test images. For performance comparison with the proposed method, Masood et al.'s method [10] is considered. As test images, the images used in Masood et al.'s method and Kodak images [25] are used. Figure 3 shows input image 'kodim03' of Kodak images [25], the image where the saturated region is clipped, and the resulting image of the proposed method. Note that clipped image is obtained by clipping the color values of the saturated regions to the value '204' $(=255 \times 0.8)$ [17] in each saturated channel. The resulting images of Masood et al.'s method [10] and the proposed method of two face images [10] and seven Kodak images are represented in Figures 4 and 5, respectively. By comparing the resulting images by existing methods and the proposed method, we can show the performance of the proposed method. In addition, Table 1 shows the peak signal-to-noise ratio (PSNR) of the clipped image, Zhang et al.'s method [9] , Xu et al.'s method [17], Masood et al.'s method [10], and the proposed method for objective assessment.

Figure 3 shows the color distortion caused by the clipping effect [17] . Figures 3(a), 1(c), and 1(e) represent the input saturated image ('kodim03'), the clipped image, and the resulting image by the proposed method, respectively. In addition, the intensity profiles along the black solid lines of Figures 3(a), 3(c), and 3(e) are shown in Figures 3(b), 3(d), and 3(f), respectively. Saturation occurs on the yellow hat where the black solid line is passed through. Figure 3(b) is the intensity profile of the correctly exposed image in Figure 3(a). On the other hand, in the Figure 3(d) which is the intensity profile of Figure 3(c) where the color values of R and B channels is saturated to '204', color saturation occurs on the yellow hat with saturation in $R$ and $B$ color values. Figure 3(f), which is the intensity profile of the proposed method, shows the corrected intensity profile preserving the original color variation. 


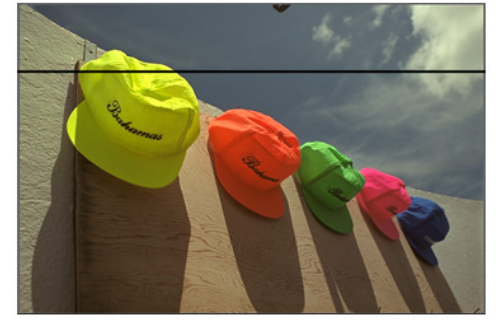

(a)

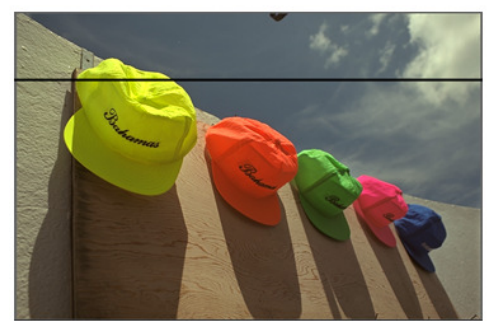

(c)

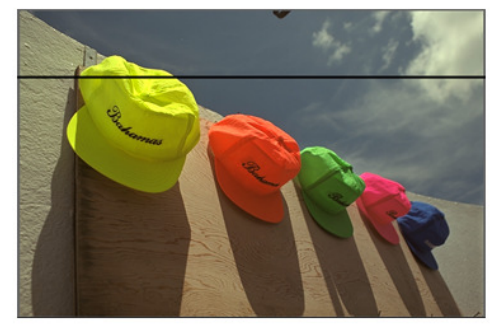

(e)

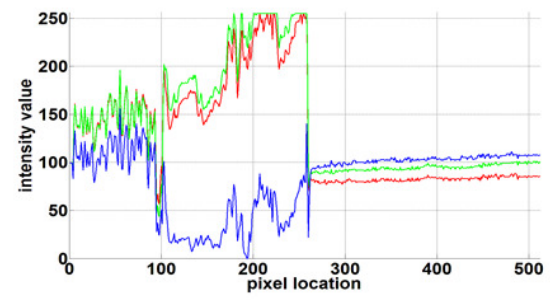

(b)

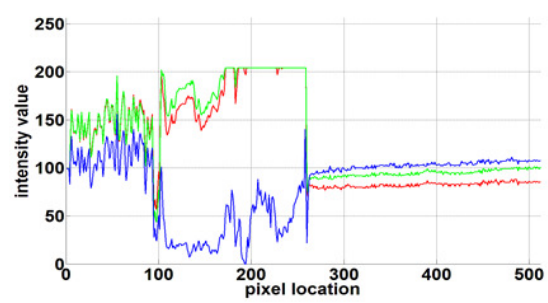

(d)

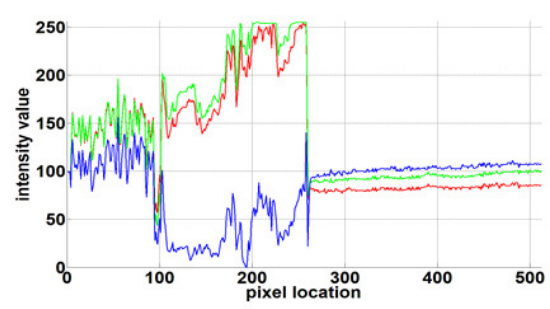

(f)

Figure 3. Input, distorted, and corrected images. (a) Input image, (b) Intensity profile along the black solid line in (a), (c) Clipped image, (d) Intensity profile along the black solid line in (c), (e) Resulting corrected image by the proposed method, (f) Intensity profile along the black solid line in (e).

Figure 4 shows the comparison of the experimental results of two face images with saturation. Figure 4(a) shows two input images and Figure 4(b) shows the images where the saturated region is represented by the color values. The clipped images having the color value '204' at the saturated pixel are shown in Figure 4(c), and the resulting images of Masood et al.'s method [10] and the proposed method are represented in Figures 4(d) and 4(e), respectively. In the case of human face, color saturation occurs by the clipping in $\mathrm{R}$ color channel and results in red colored faces. This color saturation is represented in Figure 4(a). At the boundary of the faces, the color is saturated indicating large red color and white saturated region is shown in the center of the faces with the saturated $R, G$, and $B$ color channels. In Figure 4(c), unnatural color representation with largely darker face color occurs on the faces. Additionally, in Figure 4(d) with the resulting images by Masood et al.'s method, the brightness of the whole images become darker regardless of the saturated regions by intensity scaling. Though color saturation is reduced to some degree, the incorrect resulting images become darker compared to the input images. On the other hand, in Figure 4(b) with the resulting images by the proposed method, most red color is reduced near the boundaries of the faces and the white regions in the centers of the faces also are corrected to some extent. 

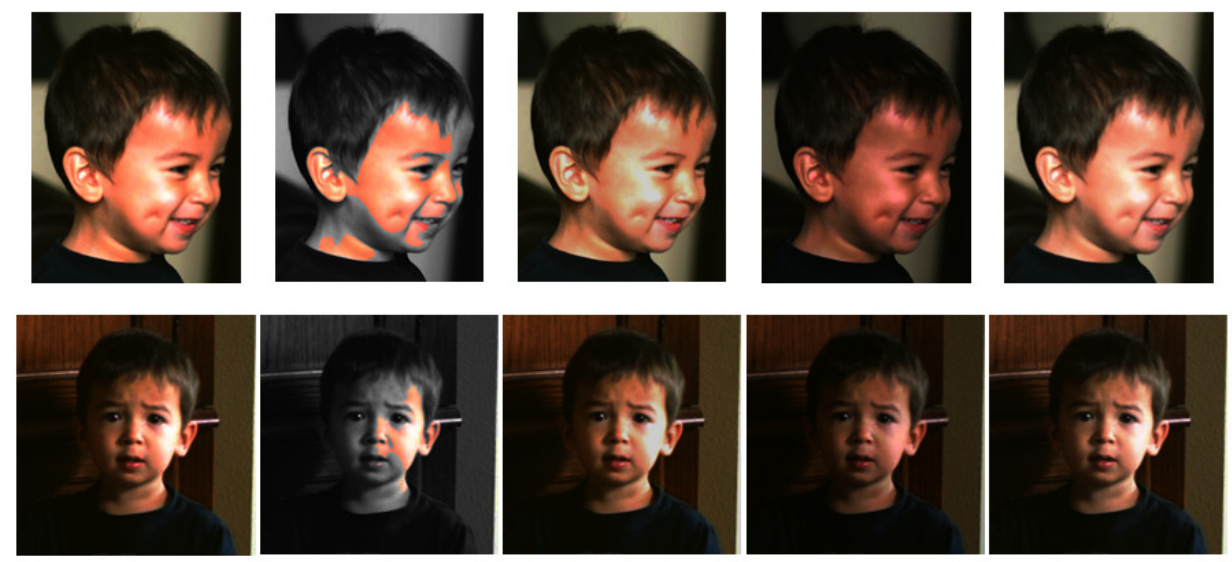

(a)

(b)

(c)

(d)

(e)

Figure 4. Input and resulting images in two saturated face images [10] . (a) Input images, (b) Images, where the saturated region is represented with color, (c) Clipped images, (d) Resulting images by Masood et al.'s method [10], (e) Resulting images by the proposed method.

In Figure 5, seven input images of Kodak images [25] as well as the resulting images by Masood et al.'s method [10] and the proposed method are shown. Figure 5(a) shows seven input images (kodim03, kodim05, kodim06, kodim12, kodim16, kodim21, and kodim23) from top to bottom row. Figure 5(b) shows the images where the saturated region is represented with the original color values and Figure 5(c) shows the clipped images. In Figures 5(d) and 5(e), the resulting images of Masood et al.'s method [10] and the proposed method are shown. In each clipped image of Figure 5(c), color distortion caused by saturation occurs with over-exposure in the saturated regions. That is, the clipped images are represented darker and more unnatural compared to the input images in Figure 5(a). In the case of the resulting images of Masood et al.'s method in Figure 5(d), the images are obtained and scaled by using auto-adjustment in Photoshop tool 'image-adjustment-curve' after saturation correction by Masood et al.'s method. After that, the whole images become darker regardless of the saturated regions. Especially, the yellow colored regions in 'kodim06' and 'kodim23' of Figure 5(d) show incorrect and unnatural color values compared to the neighboring regions. That is, the yellow hat and parrot are represented by green color. It is because $R$ value in the saturated region is excessively reduced by Masood et al.'s algorithm. Unlike the incorrect resulting images of Masood et al.'s method, in the resulting images by the proposed method shown in Figure 5(e), the saturated region is corrected naturally without artifact between the corrected and the neighboring regions.

Table 1 shows objective assessment results of seven Kodak images [25] in Figure 5. In order to perform objective assessment, the PSNR value is used, which is defined as

$$
\begin{gathered}
P S N R=10 \log _{10}\left(\frac{255^{2}}{M S E}\right), \\
M S E=\frac{1}{3 M N} \sum_{d=1}^{3} \sum_{j=0}^{N-1} \sum_{i=0}^{M-1}[I(i, j, d)-\hat{I}(i, j, d)]^{2},
\end{gathered}
$$



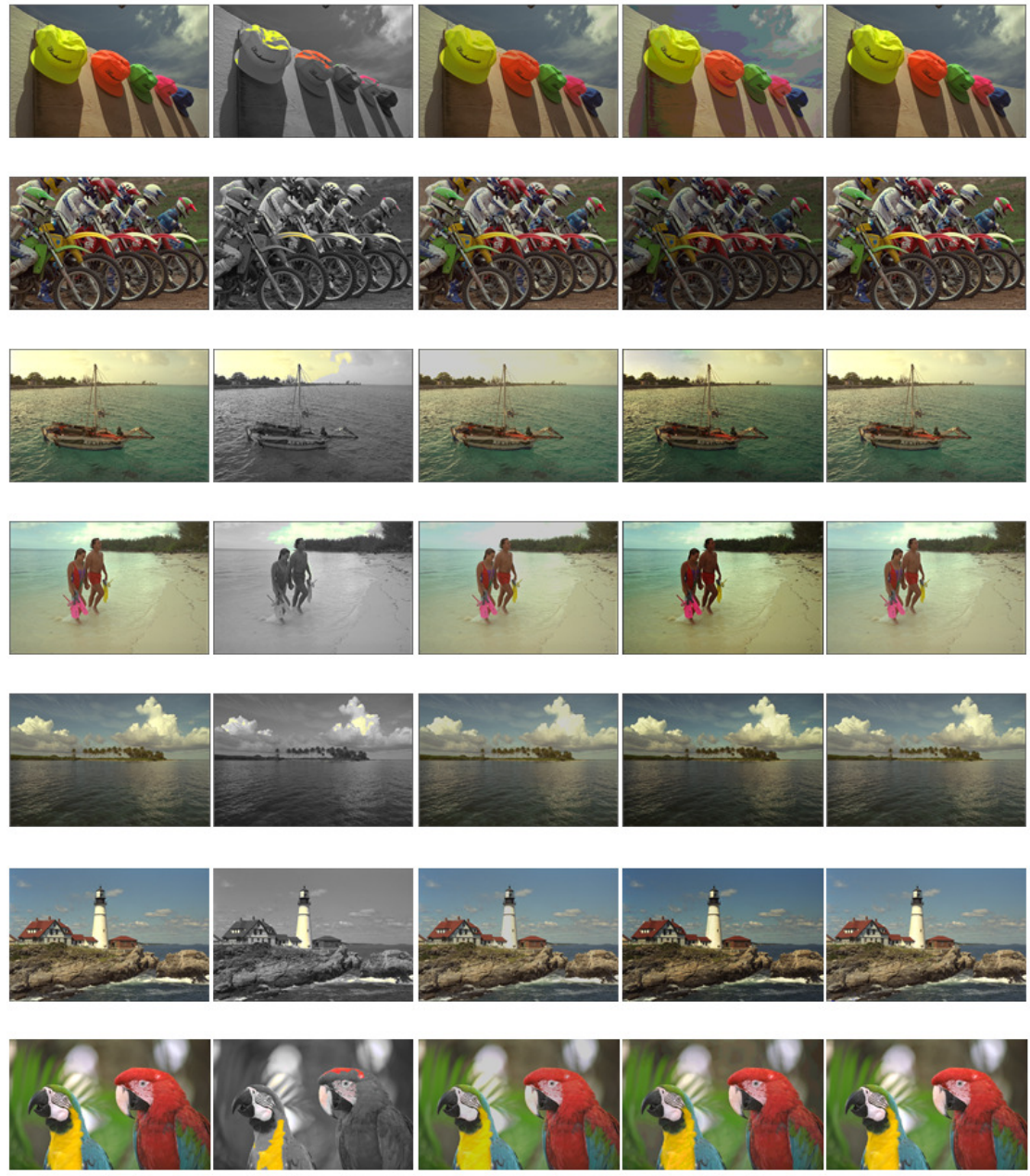

(a)

(b)

(c)

(d)

(e)

Figure 5. Input and resulting images in seven Kodak images [25] . (a) Input images, (b) Images, where the saturated region is represented with color, (c) Clipped images, (d) Resulting images by Masood et al.'s method [10], (e) Resulting images by the proposed method.

where $I$ is the input image with correct exposure, i.e., ground truth image, and $\hat{I}$ is the clipped image influenced by over-exposure or the resulting images by the existing methods [9] , [17] , [10] or the proposed method. $M$ and $N$ denote the horizontal and vertical lengths of the images and $d$ is the dimension of the color image. Clipped image is the distorted image with overexposure and the input image can be used as ground truth and criteria of the comparison. The larger the PSNR of the resulting image is, the more similar the resulting image is to the input ground truth image, because the mean square error (MSE) of the clipped image is computed using the difference between the input image and the clipped image. In addition, the PSNR is inversely proportional to the MSE. Therefore, we can conclude that the resulting image with the largest PSNR value is the best corrected image. In Table 1, Zhang and Brainard's method [9] , Xu et al.'s method [17], Masood et al.'s method [10], and the proposed method are compared in terms of the PSNR. The PSNRs of the existing methods are from respective references, therefore, the PSNR comparison should not be taken to be very precise. Compared to the PSNR values of the clipped images, the PSNR values of the resulting images by Zhang and Brainard's method, Xu et al.'s method, and the proposed method have larger values. Because of excessive correction 
of the saturated region in Zhang and Brainard's method and Xu et al.'s method, the resulting color values in the saturated region have smaller values compared to the input images. Thus, when compared to the PSNR values of the clipped images, the PSNR values of two existing methods are not largely improved. Additionally, Masood et al.'s method shows the smallest PSNR values. It is because the color values of the resulting images have small values in whole images including the saturated regions. In the proposed method, by correcting the saturated regions using the unsaturated neighboring regions with low saturation and similar color information with the saturated regions, the original colors can be corrected and preserved. Therefore, when computing the PSNR, the proposed method obtains the largest values because of the smallest MSE values. As a result, the proposed method shows the effectiveness of the performance in the view of the objective assessment.

Table 1. Comparison of the PNSR with respect to the results in Figure 5.

\begin{tabular}{|c|c|c|c|c|c|}
\hline \multirow{2}{*}{ Input images } & \multicolumn{5}{|c|}{ PSNR (dB) } \\
\cline { 2 - 6 } & Clipped image & $\begin{array}{c}\text { Zhang and } \\
\text { Brainard's } \\
\text { method [9] }\end{array}$ & $\begin{array}{c}\text { Xu et al.'s } \\
\text { method [17] }\end{array}$ & $\begin{array}{c}\text { Masood } \text { et al.'s } \\
\text { method [10] }\end{array}$ & $\begin{array}{c}\text { Proposed } \\
\text { method }\end{array}$ \\
\hline Kodim03 & 34.34 & 35.24 & 39.71 & 26.37 & 54.81 \\
\hline Kodim05 & 33.62 & 35.74 & 37.07 & 17.37 & 49.52 \\
\hline Kodim06 & 25.22 & 25.12 & 32.51 & 20.24 & 49.96 \\
\hline Kodim12 & 28.41 & 33.65 & 33.16 & 20.08 & 49.02 \\
\hline Kodim16 & 35.07 & 35.85 & 41.74 & 23.83 & 54.95 \\
\hline Kodim21 & 32.40 & 33.56 & 36.76 & 20.50 & 50.72 \\
\hline Kodim23 & 29.63 & 31.16 & 34.85 & 31.57 & 50.49 \\
\hline
\end{tabular}

\section{Conclusions}

This paper proposes a color correction algorithm of the saturated region, where the original color values are lost and the color values are saturated to the maximum values in a digital color image. The saturated region is detected independently in R, G, and B color channels and the proposed method uses RGB color values in the non-saturated neighboring regions in order to recover the original color. The reliability of the color restoration in the saturated region is obtained by calculating the weight in each neighboring pixel using RGB color, hue, saturation, and certainty map. The color in the saturated region is corrected by computing weighted sum of RGB color values using weights given to each pixel, thus the natural corrected color can be obtained by minimizing the artifacts between the corrected and the neighboring regions. Future work will focus on the performance improvement of the proposed saturated region correction algorithm by considering gradient information in order to preserve the detail components in the saturated regions.

\section{ACKNOWLEDGEMENTS}

This work was supported in part by Samsung Electronics Co., Ltd. 
International Journal of Computer Graphics \& Animation (IJCGA) Vol.6, No.2, April 2016

\section{REFERENCES}

[1] Wetzstein, G., Ihrke, I., Heidrich, W.: 'Sensor saturation in Fourier multiplexed imaging', Proc. Conf. Computer Vision and Pattern Recognition, San Francisco, CA, USA, June 2010, pp. 545-552

[2] Abel, J.S., Smith, J.O.: 'Restoring a clipped signal', Proc. Int. Conf. Acoustics, Speech, and Signal Processing, Toronto, Canada, April 1991, 3, pp. 1745-1748

[3] Regincos-Isern, J., Batlle, J.: 'A system to reduce the effect of CCDs saturation', Proc. Int. Conf. Image Processing, Lausanne, Switzerland, September 1996, pp. 16-19

[4] Perez, P., Gangnet, M., Blake, A.: 'Poisson image editing', ACM Trans. Graphics, 2003, 3, (3), pp. 313-318

[5] Meylan, L., Daly, S., Susstrunk, S.: 'The reproduction of specular highlights on high dynamic range displays', Proc. 14th Color imaging Conference, Scottsdale, AZ, USA, November 2006, pp. $333-$ 338

[6] Tan, P., Lin, S., Quan, L., Shum, H.-Y.: 'Highlight removal by illumination constrained inpainting', Proc. 19th IEEE Int. Conf. Computer Vision, Nice, France, October 2003, pp. 164-169

[7] Shen, H.-L., Cai, Q.-Y.: 'Simple and efficient method for specularity removal in an image', Applied Optics, 2009, 48, (14), pp. 2711-2719

[8] Mairal, J., Eland, M., Sapiro, G.: 'Sparse representation for color image restoration', IEEE Trans. Image Processing, 2008, 17, (1), pp. 53-69

[9] Zhang, X., Brainard, D.H.: 'Estimation of saturated pixel values in digital color imaging', Journ. Optical Society of America A, 2004, 21, (12), pp. 2301-2310

[10] Masood, S.Z., Zhu, J., Tappen, M.F.: 'Automatic correction of saturated regions in photographs using cross-channel correlation', Computer Graphics Forum, 2009, 28, (7), pp. 1861-1869

[11] Guo, D., Cheng, Y., Zhuo, S., Sim, T.: 'Correcting over-exposure in photographs', Proc. IEEE Conf. Computer Vision and Pattern Recognition, San Francisco, CA, USA, June 2010, pp. 515-521

[12] Rouf, M., Lau, C., Heidrich, W.: 'Gradient domain color restoration of clipped highlights', Proc. IEEE Conf. Computer Vision and Pattern Recognition Workshops, Providence, RI, USA, June 2012, pp. 7-14

[13] Mansour, H., Saab, R., Nasiopoulos, P., Ward, R.: 'Color image desaturation using sparse reconstruction', IEEE Int. Conf. Acoustics, Speech, and Signal Processing, Dallas, TX, USA, March 2010, pp. 778-781

[14] Elboher, E., Werman, M.: 'Recovering color and details of clipped image regions', Int. Journ. Computer Information Systems and Industrial Management Applications, 2011, 3, pp. 812-819

[15] Tomasi, C., Manduchi, R.: 'Bilateral filtering for gray and color images', Proc. IEEE Int. Conf. Computer Vision, Bombay, India, January 1998, doi: 10.1109/ICCV.1998.710815

[16] Duran, F., Dorsey, J.: 'Fast bilateral filtering for the display of high-dynamic-range images', $A C M$ Trans. Graphics, 2002, 21, (3), pp. 24-52

[17] Xu, D., Doutre, C., Nasiopoulos, P.: 'Correction of clipped pixels in color images', IEEE Trans. Visualization and Computer Graphics, 2011, 17, (3), pp. 333-344

[18] Xu, D., Doutre, C., Nasiopoulos, P.: 'Saturated-pixel enhancement for color images', Proc. 2010 IEEE Int. Symp. Circuits and Systems, Paris, France, May/June 2010, pp. 3377-3380

[19] http://www.mssl.ucl.ac.uk/surf/guides/index.html, Yohkoh Analysis Guide: Instrument Guide, accessed October 2015

[20] Gamal, A.E., Eltoukhy, H.: 'CMOS image sensors', IEEE Circuits and Devices Magazine, 2005, 21, (3), pp. 6-20

[21] Kang, S.B.: 'Automatic removal of chromatic aberration from a single image', Proc. IEEE Int. Conf. Computer Vision and Pattern Recognition, Minneapolis, MN, USA, June 2007, pp. 1-8

[22] Gonzalez, R.C., Woods, R.E.: 'Digital Image Processing' (Pearson Education Inc., Upper Saddle River, NJ, USA, 3rd edn. 2010)

[23] Lee, D.-K., Kim, B.-K., Park, R.-H.: 'Purple fringing correction using colorization in Yxy color space', 2011 Digest of Technical Papers Int. Conf. Consumer Electronics, Las Vegas, NV, USA, January 2011, pp. 489-490

[24] Xu, D., Doutre, C., Nasiopoulos, P.: 'An improved Bayesian algorithm for color image desaturation', Proc. 2010 IEEE 17th Int. Conf. Image Processing, Hong Kong, China, September 2010, pp. 1325-1328

[25] r0k.us/graphics/kodak/, accessed October 2015 